\section{Die Hysteroskopie beim Endometriumkarzinom ist sicher}

Bedenken, durch eine Hysteroskopie könnten maligne Zellen von Endometriumkarzinomen gestreut werden, sind offenbar unbegründet. Kanadische Forscher haben in einer Studie keine Hinweise darauf gefunden, dass nach solchen Untersuchungen gehäuft fortgeschrittene Tumorstadien diagnostiziert würden.

n die retrospektive Kohortenanalyse ließen Gynäkologen der Universität Calgary Daten von 1.972 Frauen einfließen, die zwischen 1997 und 2006 an einem Endometriumkarzinom erkrankt waren. 672 von ihnen $(34,1 \%)$ hatten sich vor ihrer Operation einer diagnostischen Hysteroskopie unterzogen.

Die Analyse ergab keine signifikanten Unterschiede in den Raten von intraoperativ festgestellten Tumoren im Stadium III zwischen den Frauen nach Hysteroskopie und jenen der Vergleichsgruppe (7,1 vs. $6,5 \% ; p=0,38)$. Daten von Frauen, deren Karzinome sich bereits im Stadium IV befanden, gingen nicht in die Studie ein, weil anzunehmen war, dass die ausgeprägte klinische Symptomatik keine präoperative Endometriumprobe mehr erforderte.

13,2\% der Frauen, die eine Hysteroskopie hinter sich hatten, waren in der Zwischenzeit verstorben. Von den nicht hysteroskopisch untersuchten Patientinnen waren $15,2 \%$ gestorben - dieser Unterschied war ebenfalls statistisch irrelevant $(p=0,25)$. Auch hatten sich bei den Verstorbenen keineswegs signifikant gehäuft
Tumoren vom Stadium III gezeigt, wenn sie zu Lebzeiten hysteroskopiert worden waren $(21,3$ vs. $13,7 \%$; $=0,1)$. Bei $46,1 \%$ der verstorbenen Hysteroskopierten und $42,1 \%$ der gestorbenen Frauen in der Vergleichsgruppe war der Tod auf Krebs der weiblichen Genitalorgane zurückzuführen $(p=0,53)$. Die Sorge, durch eine Hysteroskopie die präoperative Tumoraussaat bei Frauen mit Endometriumkarzinom zu fördern, ist in der Vergangenheit immer wieder geäußert worden. Tatsächlich kann sich bei Drücken von 100-150 $\mathrm{mmHg}$ gasförmiges oder flüssiges Distensionsmedium über die Zervix und die Tuben hinaus ausdehnen. Theoretisch wäre es daher denkbar, dass damit maligne Zellen in die Bauchhöhle gelangen.

Fazit: Eine präoperative Hysteroskopie bei Frauen mit Endometriumkrebs hat laut den Ergebnissen der vorliegenden Studie also keine Aussaat von Krebszellen in das Peritoneum zur Folge. Jedenfalls sind im Anschluss an die Untersuchung intraoperativ nicht häufiger fortgeschrittene Stadien der Erkrankung zu sehen. Damit darf das diagnostische
Verfahren, das eine direkte Sicht und gezielte Biopsie erlaubt, als sicher gelten.

Robert Bublak

Soucie JE et al. The risk of diagnostic hysteroscopy in women with endometrial cancer. Am J Obstet Gynecol. 2012;207(1): 71.e1-5.

Kommentar Prof. Mallmann: Wie in den derzeit gültigen S2-Leitlinien zur Diagnostik und Therapie des Endometriumkarzinoms vorgesehen, sollte ein entsprechender Verdacht auf das Vorliegen eines Endometriumkarzinoms wie z.B. eine Blutung in der

„Die alleinige Durchführung einer fraktionierten Abrasio ohne vorherige Hysteroskopie ist obsolet."

Postmenopause durch eine diagnostische Hysteroskopie in Verbindung mit einer fraktionierten Abrasio abgeklärt werden. Die alleinige Durchführung einer fraktionierten Abrasio ohne vorherige Hysteroskopie ist obsolet. Eine Risikoerhöhung durch die intraoperative Aussaat von Tumorzellen infolge des Austretens von Flüssigkeit über die Tuben ist durch eine Vielzahl von Studien, wie auch der vorliegenden Untersuchung, ausgeschlossen.

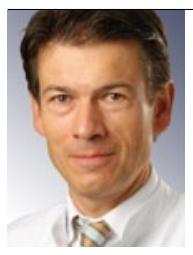

Prof. Dr. med.

Peter Mallmann Klinik und Poliklinik für Frauenheilkunde und Geburtshilfe, Universität zu Köln peter.mallmann@uk-koeln.de

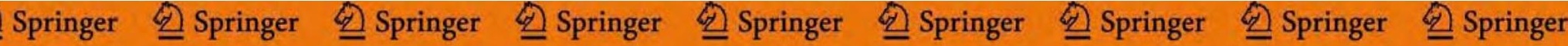

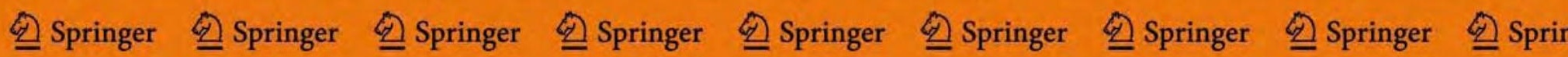

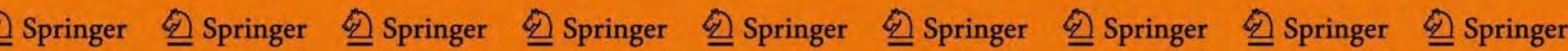
은

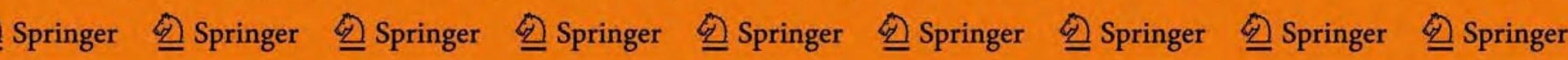

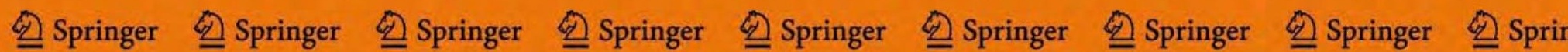

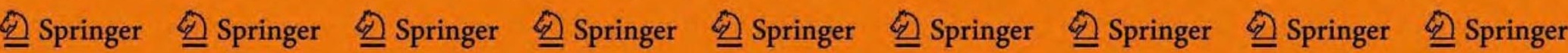

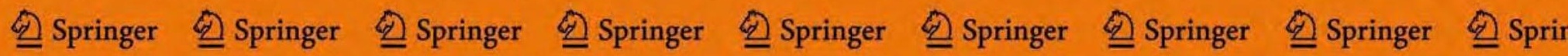

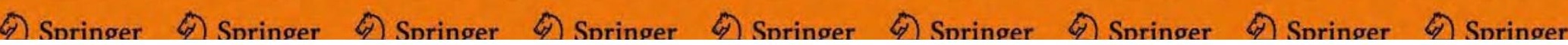

